\title{
Pharmacogenetics; mini review
}

\begin{abstract}
The extent of genetic variations found in drug metabolism genes and its contribution to inter-individual variation in response to medication remains incompletely understood. Pharmacogenetics is study of the role of inheritance in inter-individual variation in drug response. Different factors can influence the drug metabolism which in turn changes the response of drugs. The topic provides the overview the role of genetic variations that can affects the drug response. Cytochrome P450 enzyme system is super family of metabolizing enzymes, responsible for metabolism of many clinically used drugs, biosynthesis and degradation of many endogenous substances. Metabolism is generally occurs in two phases, phase I and phase II reactions. CYP450 is mostly involved in phase I reactions. The enzyme system is involved in approximately $80 \%$ of oxidative metabolism of drugs and $50 \%$ of overall elimination of drugs.
\end{abstract}

Keywords: pharmacogenetic, genetic polymorphism, drug metabolism, cytochrome p450 enzyme system
Volume 7 Issue 2 - 2018

\author{
Shumaila Arshad, Juawairiya Butt, Riaz \\ Ahmed, Muhammad ljaz \\ The University of Lahore, Pakistan
}

Correspondence: Shumaila Arshad, Faculty of Pharmacy, The University of Lahore, Lahore, Pakistan, Tel +92 3005898736, Email shumailapharmacist@gmail.com

Received: December 26, 2017 | Published: March 21, 2018

\section{Introduction}

If it were not for the great variability among individuals medicine might as well be a science and not an art." The thoughts of Sir William Osler in 1892 reflect the view of medicine over the past 100 years. ${ }^{1}$ Modern pharmacogenetics has its origins in the $1950 \mathrm{~s}$ with a series of discoveries. ${ }^{2}$ The genetic basis underlying variations in drug response has become evident with the introduction of modern analytical methods for analysis of gene sequence and expression. Pharmacogenetics is the study of how the genes affect the way people responds to therapy. There is great heterogeneity in the way people respond to the medications in term of toxicity and treatment efficiency. ${ }^{3}$ The variations in response may be due to environmental factors or genetic factors. The environmental factor which influences the response can be other drugs, nutritional components, smoking or liver disease; they can cause the difference in activity either by inducing or inhibiting the drug metabolizing enzymes. In last few years, it has been realized that common genetic polymorphism of metabolizing enzymes can affect the response of individuals towards drugs. Genetic polymorphisms of drug metabolizing enzymes give rise to distinct subgroups in the population which differs in their ability to perform a certain drug biotransformation reactions. The goal of Pharmacogenetics is to individualize drug therapy to a person's unique genetic makeup. ${ }^{4}$ Genetic makeup determines the inherent pharmacokinetics, which shows the interpersonal differences in drug absorption, distribution, metabolism and elimination. These differences can be explained by genetic variation in transport proteins, drug targeting, and in function of cytochrome P450 or phase 2 drug metabolizing enzymes.

Research in pharmacogenetics is currently developing in two main directions: firstly, identifying specific genes and gene products associated with various diseases, which may act as targets for new drugs, and, secondly, identifying genes and allelic variants of genes that affect our response to current drugs. ${ }^{5}$ Pharmacogenomics is closely related term which considered to overlapping field. It can be defined as the study of the role of genes and their genetic variations in the molecular basis of disease, and therefore influence or change the pharmacological effect of drugs on the disease. Pharmacogenetics and
Pharmacogenomics are both important in the study of genes that encode for drug metabolizing enzymes, drug receptors, drug transporters and ion channels or efflux system. The term polymorphism means that a character appears in classifiable forms among individual due to the difference in alleles present at a single gene locus. To appear polymorphic, the metabolism must show high degree of specificity for enzymes that exhibits genetic polymorphism. If it is not showing specificity the rate of metabolism will not show separate distribution into categories of different capacities, even if it is polymorphic for particular enzyme. Genes are considered polymorphic with a major allele encoding the normal protein, and one or more minor alleles with alteration in functions. Due to these alterations, the result is either reduction in function, or absence of function and in some cases there is increase in function. Pharmacogenomics techniques allow efficient analysis of the risk factors, and genotyping tests have the potential to optimize drug therapy in the future. ${ }^{6}$ The review mainly highlights the genetic variations in cytochrome P450-mediated drug metabolism. ${ }^{4,7,8}$

\section{Biotransformation or metabolism}

Pharmacogenetics encompasses the involvement of genes in an individual's response to drugs. ${ }^{9}$ The process of metabolism or biotransformation is the enzymatic conversion of a drug to a metabolite. The enzymes play an important role in the inactivation and subsequent elimination of drugs that are not easily cleared through the kidney. For few drugs metabolism results in the formation of more polar metabolites that are pharmacologically inactive and eliminated more rapidly than the parent drug. For some drugs the metabolites may be pharmacologically active or produce toxic effects. These drugs must be metabolized or biotransformed in the body to metabolites that have pharmacologic activity. Individual with deficiency in metabolic activity, as compared to normal extensive metabolizer or EM-phenotype, are known as poor metabolizer or PM-Phenotypes. ${ }^{10}$

Phenotype is the observed characteristic of a patient's enzyme activity, and includes such designations as "poor metabolizer," "intermediate metabolizer," "extensive metabolizer," and "ultra rapid extensive metabolizer." Patients who express dysfunctional or inactive enzymes are considered poor metabolizer. The overall pharmacological effects are not monogenic traits; they can be 
determined by the interplay of several genes encoding proteins which are involved in multiple pathways of metabolism. The polymorphic drug metabolizing enzymes that exhibits the co-dominant inheritance determines the plasma concentrations to which every individual is exposed, and the polymorphic receptor determines the nature of response at any concentration of drug. The drug toxicity can be determined by receptors that do not show functionally important genetic polymorphism or by the genetic polymorphism in the drug receptors. Thus the individuals with homozygous wild type drug metabolizing enzymes and drug receptor would have a high chance of therapeutic efficacy and low toxicity as compared to those having homozygous mutant genotypes for drug metabolizing enzymes and receptors. ${ }^{3}$

Drug metabolism generally occurs in two chemical processes, i.e. phase-I reactions involving oxidation, reduction and hydrolysis and phase-II reactions which included glucuronidation, sulfation, acetylation, and methylation. ${ }^{11}$ The phase-I reactions occur first and introduce or expose a functional group on the drug molecules. In phase-II reactions those drug molecules form conjugates with endogenous substances such as glucuronic acid. The acetylation is important conjugation reaction because the acetylated product is usually less polar than the parent drug.

Variation in drug metabolism must be studied to reflect variable amount of accessible enzymes, difference in their molecular structure or effect of modifiers on enzymes activity. The amount of the enzyme available may be subjected to temporary alteration such as enzymes induction, structural variation is difficult to investigate in the mixedfunction oxidase system because the enzyme system contains multiple forms of cytochrome P450. Every form of enzyme system is expected to have some genetic variation. The enzyme activity can be altered due to the presence of any inhibitor or activator. State of lipid oxidation can also affect the activity of membrane bound enzymes. Other than this, the activity of enzymes can be altered or modify due to reduction in blood flow, membrane permeability or nonspecific binding. ${ }^{8,12}$

\section{Genetic polymorphism in drug metabolism: acetylation}

The polymorphism of N-Acetyltransferase is one of known genetic defect in drug metabolism. The first population study of Isoniazid (INH) was presented by Price Evans et al. ${ }^{12}$ The first review of acetylation Pharmacogenetics was published by Weber in 1973, which deals with acetylation variations in humans and animals i.e. inherited variations, mechanism of enzyme drug acetylation and properties that affect the acetylation of drugs. INH drug is mostly used for specific treatment of tuberculosis in children and adults. As Evans et al. ${ }^{3}$ first describes bimodality distributed concentration of INH in blood samples taken at fixed intervals after administration INH. Initial metabolic experiments showed that the human hereditary acetylation polymorphism is a major determinant of INH disposition and elimination..$^{12,13}$ The acetylation phenotypes slow and rapid may influence drug efficacy and chances of adverse reactions, can also be associated with disease state. Slow acetylators are at high risk of developing neuropathy due to INH therapy as compared to rapid acetylators. ${ }^{14}$

Family studies verify the genetic control over this variation and showed that the ability to eliminate INH was determined by 2 alleles at a single autosomal gene locus which controls the rate of $\mathrm{N}$-acetylation activity of enzyme, occurs predominantly in liver with slow acetylators. ${ }^{12}$ The variations in the rate of acetylation of INH accounts for phenotypic differences between rapid and slow acetylator. INH is converted to acetylisoniazid more rapidly in rapid acetylators than in slow acetylators. In both types of acetylators the difference occurs in INH and acetylisoniazid plasma concentrations and urinary excretion. The acetylisoniazid is metabolized in isonicotinic acid and monoacetylhydrazine (MAH). The further conjugation of isonicotinic acid is not related to acetylators' status. The MAH is acetylated to diacetylhydrazine. Rapid acetylators acetylates greater fraction of MAH as compared to slow acetylators. ${ }^{8}$

\section{Genetic polymorphism in drug metabolism: drug oxidation}

There are two completely recognized polymorphisms in drug oxidation one is Debrisoquine/sparteine and the second is S-Mephenytoin hydroxylation polymorphisms. The metabolism of some important drugs is cosegregate with Debrisoquine. The Pharmacogenetics in drug oxidation was greatly studied in mid 1970s when it was observed that the metabolism of antihypertensive agent Debrisoquine and the oxytocic agent sparteine exhibits the apparent polymorphism which reflects the monogenic control of one cytochrome P-450 isoenzyme. ${ }^{15}$ The different types of oxidative reactions which are affected by polymorphism showed the genetic variation in cytochrome P-450 isoenzyme. During 1975 to 1977 two different groups separately pioneer the polymorphism in Debrisoquine and sparteine metabolism. One group investigated Debrisoquine metabolism which was due to impaired 4-hydroxylaton of Debrisoquine. Second group at the same time investigated increased side effects related with decreased oxidative metabolism of sparteine which is an oxytocic and antiarrhythmic alkaloid. ${ }^{16}$ Hammer and Sjoqvist studied 36-fold variation in concentration after observing the 11 patients who was treated with a standard dose of demethylimipramine which is an antidepressant drug. They observed that the metabolism of drug was analogous with the Debrisoquine hydroxylation polymorphism. So it was expected that the drug metabolic polymorphism increase the drug clearance variations in different individuals.Family studies shown that the poor metabolizers are homozygous for a recessive gene and the rapid metabolizers can be both homozygous and heterozygous for dominant gene. It was marked that the two deficient reactions were identical or linked by genetic control; a poor metabolizer of Debrisoquine is always a poor metabolizer of sparteine and similarly the poor metabolizer of sparteine is always a poor metabolizer of Debrisoquine. In last few years it has been revealed that poor metabolizers also show an impaired capacity for the oxidative metabolism of about 20 other drugs including $\beta$-adrenergic blocking agents, antiarrhythmics, antidepressants, opioids and several other drugs. Clinical studies confirmed that poor metabolizers of Debrisoquine and other above mentioned drugs signify a high risk group in the population with a tendency to extend adverse drug effects. ${ }^{16}$

Mephenytoin an antiepileptic drug was introduced into clinical use in 1945. The polymorphism of hydroxylation of S-Mephenytoin was not well studied as compare to Debrisoquine hydroxylation. The metabolic reactions which are catalyzed by polymorphic enzymes are extremely fastidious for the $\mathrm{S}$ form of the racemic Mephenytoin. TheMephenytoin is now been neglected and Diazepam, Omeprazole, proguanil and chlorproguanil are most frequently used. The final compounds are reliant on metabolic activation markedly catalyzed by the Mephenytoin hydroxylase. The cytochrome catalyzing Mephenytoin hydroxylation is subfamily P-45OIIC. ${ }^{17}$ 


\section{Cytochrome P450 system}

The field of cytochrome P450 pharmacogenetics has progressed rapidly during the past 25 years. ${ }^{18}$ Cytochrome P450 enzymes system is super family of microsomal drug metabolizing enzymes, which is located in the endoplasmic reticulum of hepatic cells. Their characteristics are well established and their involvement in metabolism of different drugs is also well known. As the metabolism occurs in two phases, these enzymes are mostly involved in phase I reactions. ${ }^{19}$ These enzymes are responsible for biosynthesis and degradation of endogenous compounds such as steroids, lipids and vitamins. They have ability to reduce or change the pharmacological effect of drugs, Cytochrome P450 enzymes are notorious for their ability for changing the response of drug by oxidative or reductive reactions. ${ }^{11,20}$ Many variations in drug response are recognized due to occurrence of adverse reactions after standard dose of drug in patients. $^{21}$

To add standardization to the CYP450 system, the nomenclature system was adopted in 1996 for the classification of enzyme system. The nomenclature is based on amino-acid identity, phylogenetic criteria and gene association. To exemplify this naming system, take CYP2C19*2*3 as an example. In this isoenzyme designation, CYP is the standard abbreviation for mammalian cytochrome P450. These isoenzymes share greater than $40 \%$ protein sequence homology with each other and are nominated by the first number following the cytochrome P450 designation (e.g., 2). Subfamilies share greater than 55\% homology with each other and are differentiated by the letter following the family designation (e.g., C). Single members of subfamily represent a particular gene and are nominated by the number following the subfamily description (e.g., 19). An asterisk and a number following the member explanation assign an allele (e.g., *2 and $* 3$; two alleles since inheritance of genetic code dictate resultant drug-metabolizing enzyme activity follows Mendelian genetics). The *2 allele for CYP2D6, and most others, is known as the wild type and denotes normal enzyme activity. ${ }^{11,22,23}$

Humans consists 57 cytochrome P450 functional genes. Most of the genes are grouped according to their sequence similarities, into 18 families and 44 subfamilies. They are having specific endogenous functions including biosynthesis of steroids hormones, bile acid and other compounds. The CYP1, CYP2, and CYP3 families are main contributors in drug metabolism of many drugs and xenobiotics. ${ }^{11,24}$ The enzyme system is involved in approximately $80 \%$ of oxidative metabolism of drugs and $50 \%$ of overall elimination of drugs. As CYP450 enzymes are having specific unique substrate, they are involved in particular drug molecules metabolism but over lapping may also present. So a single isoenzyme may contribute in metabolism of large number of drugs or more than one isoenzyme may involve in metabolism of one drug. ${ }^{11}$

Cytochrome P450s are divided in four major classes based on how electrons are transferred to catalytic site from Nicotinamide adenine dinucleotide phosphate (NADPH). Class-I proteins entail both flavin adenine dinucleotide (FAD) containing reductase and an iron sulfur redoxin for electron transfer. Class-II proteins entail only FAD/Flavin mononucleotide (FMN) containing P450 reductase for transfer of electrons. Class-III enzymes are self-reliant and need no electron donor. Class-IV receives electrons from Nicotinamide adenine dinucleotide phosphate directly. ${ }^{23}$ The substrate molecules can interact with cytochrome isoenzymes in a similar pattern as drug binding with receptor. For that reason drugs and their metabolites act as specific substrate for specific isoenzymes and then these isoenzymes can be blocked, induced or remain active in presence of other drug. Any molecule that diminishes the activity of Cytochrome P450 enzymes pathway, is known be an inhibitor of enzymes. Different environmental chemicals or drugs can increase the enzymes activity by increasing mRNA concentration of isoenzyme by means of increased gene expression, and known to be an inducer for enzyme. ${ }^{4}$

\section{Conclusion}

Pharmacogenetic is study of inherited genetic differences in metabolic pathways that can affect the response of drug in every individual. The variation in the genes which are responsible for metabolism of drug plays an important role in the drug's pharmacological effect. Pharmacogenetic is new topic in the medical filed which have huge impact on almost all medical issues including treatment of both common and rare disease.

\section{Acknowledgements}

None.

\section{Conflict of interests}

The author declares that there is no conflict of interest.

\section{References}

1. Roses AD. Pharmacogenetics and the practice of medicine. Nature. 2000;405(6788):857-865.

2. Gardiner SJ, Begg EJ. Pharmacogenetics, drug-metabolizing enzymes, and clinical practice. Pharmacol Rev. 2006;58(3):521-590.

3. Evans WE, Relling MV. Pharmacogenomics: translating functional genomics into rational therapeutics. Science. 1999;286(5439):487-491.

4. Ereshefsky L, Dugan D. Review of the pharmacokinetics, pharmacogenetics, and drug interaction potential of antidepressants: focus on venlafaxine. Depress Anxiety. 2000;12(1):30-44.

5. Wolf CR, Smith G, Smith RL. Science, medicine, and the future: Pharmacogenetics. BMJ. 2000;320(7240):987-990.

6. Meyer UA. Pharmacogenetics and adverse drug reactions. Lancet. 2000;356(9242):1667-1671.

7. Ghotbi R, Gomez A, Milani L, et al. Allele-specific expression and gene methylation in the control of CYP1A2 mRNA level in human livers. Pharmacogenomics J. 2009;9(3):208-217.

8. Rios-Santos F, Magno LAV. Pharmacogenetics and metabolism: past, present and future. Topics on drug metabolism. Rijeka: InTech Europe. 2012. p. 61-86.

9. Spear BB, Heath-Chiozzi M, Huff J. Clinical application of pharmacogenetics. Trends Mol Med. 2001;7(5):201-204.

10. Draganov D, La Du B. Pharmacogenetics of paraoxonases: a brief review. Naunyn-Schmiedebergs arch pharmacol. 2004;369(1):78-88.

11. Wilkinson GR. Drug metabolism and variability among patients in drug response. N Engl J Med. 2005;352(21):2211-2221.

12. Crettol S, Petrovic N, Murray M. Pharmacogenetics of phase I and phase II drug metabolism. Curr phar Des. 2010;16(2):204-219.

13. Yamada S, Richardson K, Tang M, et al. Genetic variation in carboxylesterase genes and susceptibility to isoniazid-induced hepatotoxicity. Pharmacogenomics J. 2010;10(6):524-536. 
14. Sim E, Abuhammad A, Ryan A. Arylamine N-acetyltransferases: from drug metabolism and pharmacogenetics to drug discovery. $\mathrm{Br} J$ Pharmacol. 2014;171(11):2705-2725.

15. Pilgrim J, Gerostamoulos D, Drummer OH. Review: Pharmacogenetic aspects of the effect of cytochrome $\mathrm{P} 450$ polymorphisms on serotonergic drug metabolism, response, interactions, and adverse effects. Forensic Sci Med Patholo. 2011;7(2):162-184.

16. El Gendy MA, Ali BH, Michail K, et al. Induction of quinone oxidoreductase 1 enzyme by Rhazya stricta through Nrf2-dependent mechanism. J Ethnopharmacol. 2012;144(2):416-424.

17. Thorn CF, Whirl-Carrillo M, Leeder JS, et al. PharmGKB summary: phenytoin pathway. Pharmacogenet Genomics. 2012;22(6):466-470.

18. Ingelman Sundberg M. Pharmacogenetics of cytochrome P450 and its applications in drug therapy: the past, present and future. Trends Pharmacol Sci. 2004;25(4):193-200.
19. Solus JF, Arietta BJ, Harris JR, et al. Genetic variation in eleven phase I drug metabolism genes in an ethnically diverse population. Pharmacogenomics. 2004;5(7):895-931.

20. Samer CF, Lorenzini KI, Rollason V, et al. Applications of CYP450 testing in the clinical setting. Mol Diagn Ther. 2013;17(3):165-184.

21. Zhou HH, Liu ZQ. Ethnic differences in drug metabolism. Clinical chemistry and laboratory medicine. 2000;38(9):899-903.

22. Rogers JF, Nafziger AN, Bertino JS. Pharmacogenetics affects dosing, efficacy, and toxicity of cytochrome P450-metabolized drugs. Am J Med. 2002;113(9):746-750.

23. Werck-Reichhart D, Feyereisen R. Cytochromes P450: a success story. Genome Biol. 2000;1(6).

24. Zanger UM, Schwab M. Cytochrome P450 enzymes in drug metabolism: regulation of gene expression, enzyme activities, and impact of genetic variation. Pharmacol Ther. 2013;138(1):103-141. 